\title{
An EnKF-based Flow State Estimator for Aerodynamic Flows
}

\author{
Andre F. C. da Silva* and Tim Colonius ${ }^{\dagger}$ \\ California Institute of Technology, Pasadena, CA, 91125, United States of America
}

\begin{abstract}
Regardless of plant model, robust flow estimation based on limited measurements remains a major obstacle to successful flow control applications. Aiming to combine the robustness of a high-dimensional representation of the dynamics with the cost efficiency of a low-order approximation of the state covariance matrix, a flow state estimator based on the Ensemble Kalman Filter (EnKF) is applied to two-dimensional flow past a cylinder and an airfoil at high angle of attack and low Reynolds number. For the development purposes, we use the numerical algorithm as both the estimator and as a surrogate for the measurements. Estimation is successful using a reduced number of either pressure sensors on the surface of the body or sparsely placed velocity probes in the wake. Because the most relevant features of these flows is restricted to a low-dimensional subspace/manifold of the state space, asymptotic behavior of the estimator is shown to be achieved with a small ensemble size. The relative importance of each sensor location is evaluated by analyzing how they influence the estimated flow field. Covariance inflation is used to enhance the estimator performance in the presence of unmodeled free stream perturbations. A combination of parametric modeling and augmented state methodology is used to successfully estimate the forces on immersed bodies.
\end{abstract}

\section{Introduction}

The agility, endurance and maneuverability of the next-generation aircraft could be enhanced if they could tolerate a broad spectrum of gusts while safely performing maneuvers that would otherwise lie outside of the flight envelope. Closed-loop control techniques might yet achieve these goals, but a pacing item in deploying them are techniques to robustly estimate the flow state from the available measurement data such as pressure readings on the wings and fuselage.

Although several state estimation techniques have been developed, their application to fluid dynamics is challenging due to the nonlinearity and high dimensionality of the underlying physical phenomena. Fluid systems are represented by spatially continuous models and any suitable discretization results in highdimensional discrete models. Whereas feedback control applications requires real-time state estimation, the computational cost of standard control techniques such as the Kalman filter ${ }^{1}$ do not scale well with increasing flow complexity and faster time scales. Also, numerical simulations require appropriate boundary and initial conditions that are uncertain. ${ }^{2}$ Measurement data can provide the necessary information to close the gap between simulation and experiments. The development of methodologies for the seamless integration of measurement data and (often sophisticated) mathematical models is the goal of a research area known as data assimilation.

Within the flow control community, the most common approach to reduce computational cost is the development of reduced-order models (ROMs). Provided these models have a low number of degrees of freedom, then the classical control techniques becomes feasible. Ahuja and Rowley ${ }^{3}$ used a 22-mode ROM obtained by Balanced Truncation to design a LQG controller for the flow past an inclined flat plate. Flinois and Morgans ${ }^{4}$ used the Eigenvalue Realization Algorithm (ERA) to construct an unstable 10-mode ROM which was then used to design $\mathcal{H}_{\infty}$ controllers to stabilize the system. These ROMs, however, are fragile with respect to initial conditions and flow parameters like the Reynolds number. Alternatively, researchers such

\footnotetext{
*Graduate Student, andrefcs@caltech.edu.

${ }^{\dagger}$ Professor of Mechanical Engineering, AIAA Associate Fellow.
} 
as Fukumori \& Malanotte-Rizzoli ${ }^{5}$ and Suzuki $^{6}$ proposed the use of reduced-order approximations to the Kalman filter that restrict the correction to the larger scales of the solution and alleviate the computational cost involved.

On the other hand, researchers in fields such as meteorology, oceanography and geophysical fluid dynamics have developed data assimilation algorithms that are inherently capable of dealing with high-dimensional nonlinear systems and high volumes of measurement data. ${ }^{7,8}$ These methods take full advantage of the increasingly available computational power and efficient parallel implementations, and had not, until recently, received much attention from the flow control community. Examples include the work by Colburn et al., ${ }^{9}$ in which a Ensemble Kalman Filter (EnKF) was applied to the problem of estimating the flow in a turbulent channel flow, and by Kikuchi et al., ${ }^{10}$ where both the EnKF and a Particle Filter were used in conjunction to a POD-Galerkin method for the problem of the flow past a cylinder.

A well-known alternative for sequential data assimilation of high dimensional systems is the $3 \mathrm{D}$-Var. ${ }^{11}$ Just like the Kalman filter, 3D-Var can be formulated as an observer in which the gain is calculated to minimize a cost function, with the general format

$$
J(x)=\left[y_{0}-h(x)\right]^{T} R^{-1}\left[y_{0}-h(x)\right]+\left[x-x_{f}\right] \Sigma^{-1}\left[x-x_{f}\right],
$$

where $y_{0}$ is the measurement taken from the tracked system, and $x_{f}$ is the prior estimate for the state. The main difference is that 3D-Var regards $\Sigma$ as a predefined constant weight matrix, while the EnKF regards it as an ensemble-based approximation to the true state covariance matrix. Since just a single estimate must be propagated, 3D-Var is far less computationally demanding than EnKF. However, estimator performance depends heavily on the a priori choice of $\Sigma$. The EnKF is irrefutably sub-optimum in the presence of nonlinearities, but Kalman's rule provides at least a consistent way of estimating $\Sigma$ on the fly by taking into account relevant information about the dynamics.

In this paper, we employ an EnKF to estimate the state of flow past a body from pressure measurements on the surface or sparse velocity measurements in the flowfield. In section II, a brief description of the algorithm is presented. In section III, we show some preliminary results of the application of the method to the flow past a circular cylinder and a NACA 0009 airfoil at high angle of attack. Finally, section IV enumerates some conclusions.

\section{The Ensemble Kalman Filter}

Consider a input/output discrete-time system modeled by

$$
\begin{aligned}
x_{k+1} & =f_{k}\left(x_{k}, u_{k}\right)+g_{k}\left(x_{k}\right) \eta_{k}, \\
y_{k} & =h\left(x_{k}\right)+\omega_{k},
\end{aligned}
$$

where $f_{k}$ is a general function of the $n$-dimensional state $x_{k}$ and the control input $u_{k}, \omega_{k}$ accounts for state disturbances and process noises, $h(x)$ is the measurement function ( $y_{k}$ is a $p$-dimensional vector) and $\nu_{k}$ represents sensor noise. The subscript $k$ refers to quantities taken at time $t=t_{k}$. We also assume both $\eta_{k}$ and $\omega_{k}$ are zero-mean, Gaussian, and white random processes $\left(\eta_{k} \sim N\left(0, Q_{k}\right)\right.$ and $\left.\omega_{k} \sim N\left(0, R_{k}\right)\right)$ that are uncorrelated in time $\left(E\left[\eta_{k} \eta_{l}^{T}\right]=Q_{k} \delta_{k l}\right.$ and $\left.E\left[\omega_{k} \omega_{l}^{T}\right]=R_{k} \delta_{k l}\right)$.

The goal of the filtering process is to use measurement data to construct an estimate of the state $u_{k}$, denoted by $\hat{u}_{k}$, which is optimal in the sense that it minimizes the estimation variance (or, equivalently, maximize the likelihood). ${ }^{12}$ The fundamentals of optimal filtering were derived by Kalman and Bucy in 1961. ${ }^{1}$ The classical Kalman filter (KF) provides the optimum solution for the state estimation of linear systems under Gaussian-distributed noise. Within this context, the estimate for the state $x$ is regarded as a Gaussian-distributed random variable with mean $\hat{x}$ and covariance $\Sigma$.

Devising an optimal state estimator for systems exhibiting nonlinear dynamics using measurement data that is a nonlinear function of the state is more challenging. Rigorous solutions to the estimation problem for nonlinear systems are typically either narrow in applicability or computationally infeasible. ${ }^{13}$ For weakly nonlinear cases, the extended Kalman filter $(\mathrm{EKF}),{ }^{14}$ is considered the standard technique. Nevertheless, its robustness and reliability is impaired by the linearization process, and Julier and Uhlmann ${ }^{15}$ showed that even the near-ubiquitous nonlinear transformation from polar to Cartesian coordinates is enough to yield significant deviations in tracking the correct state. For cases where there are strong nonlinearities, the Unscented Kalman Filter tends to deliver better results. ${ }^{16}$ For all these KF variants, the cost of the 
propagation of the covariance matrix $\Sigma$ is in the order of $n$ evaluations of the forecast operator and becomes quickly prohibitive for large systems.

Under nonlinear dynamics, the Probability Density Function (PDF) of the estimate need not remain Gaussian, and the first two moments cease to fully represent the state. Instead, the full PDF could, in principle, be propagated through time using a suitable discretization of the Fokker-Plank equation yielding a class of schemes known as Particle Filters. ${ }^{9,17}$ Because they rely on the direct sampling of a $n$-dimensional state space, the curse of dimensionality ${ }^{18}$ is severe for these techniques, and they are only computationally tractable for systems of reduced dimension.

Aiming at overcoming the cost barrier, in 1994 Evensen $^{19}$ proposed a Monte Carlo approximation to the $\mathrm{KF}$ in which the internal state of the estimator is represented by an ensemble of particles so that the corresponding ensemble mean and covariance matrix are used to approximate $\hat{x}$ and $\Sigma$. This method was named Ensemble Kalman Filter (EnKF) and, since then, has been extensively used for high-dimensional systems (thousands of degrees of freedom or more) associated with a computationally onerous forecast (as in meteorology, oceanography and geophysical flows ${ }^{20-22}$ ). In such context, this technique has shown to provide a correct estimate of the first two moments of state of the system even for a small ensemble size (provided that the Gaussian assumption appears to hold). ${ }^{23}$

Consider a ensemble of $q$ initially independent states. The expected value of the system state correspond to the ensemble average of these states

$$
\bar{x}_{k}=\frac{1}{q} \sum_{j=1}^{q} \hat{x}_{k}^{j} .
$$

Defining the error matrix $E_{k}^{s}$ as

$$
E_{k}^{s}=\left[\hat{x}_{k}^{1}-\bar{x}_{k} \cdots \hat{x}_{k}^{q}-\bar{x}_{k}\right]
$$

and the output error matrix $E_{k}^{o}$ as

$$
E_{k}^{o}=\left[\hat{y}_{k}^{1}-\bar{y}_{k} \cdots \hat{y}_{k}^{q}-\bar{y}_{k}\right],
$$

where $\hat{y}_{k}^{j}=h\left(\hat{x}_{k}^{j}\right)$ and $\bar{y}_{k}$ is the ensemble average of the outputs, one can finally compute the ensemble covariance matrix

$$
\Sigma_{k}=\frac{1}{q-1} E_{k}^{s}\left(E_{k}^{s}\right)^{T}
$$

which is an estimate of the state covariance matrix.

The filtering process can be synthesized in two steps: ${ }^{13,24}$

- Dynamic update (or Forecast Step): The state of each ensemble member at the next time step is estimated using the (possibly nonlinear) dynamic model (Eq. 2a):

$$
\hat{x}_{k+1 \mid k}^{j}=f\left(\hat{x}_{k \mid k}^{j}, u_{k}\right)+g\left(\hat{x}_{k \mid k}^{j}\right) \eta_{k}^{j} .
$$

- Measurement update (or Analysis Step): the ensemble members are corrected in order to minimize the error with respect to the measurements in the presence of noise and model uncertainties. An efficient way to implement the measurement update step is by using the representers' formulation proposed by Evensen: ${ }^{25}$

$$
\hat{x}_{k+1 \mid k+1}^{j}=\hat{x}_{k+1 \mid k}^{j}+\Sigma_{k+1} H_{k+1}^{T} b_{k+1}^{j},
$$

where the columns of $\Sigma_{k+1} H_{k}^{T}$ are called the representers and represent the influence vectors for each measurement. The vector $b_{k}^{j}$ then represents the magnitude by which each of the representers actuates in $\hat{x}$. It can be obtained as the solution to the linear system:

$$
\left(H_{k} \Sigma_{k} H_{k}^{T}+R_{k}\right) b_{k}^{j}=y_{k}^{j}-h\left(x_{k}^{j}\right) .
$$

Each $y_{k}^{j}$ must be independently sampled from a normal distribution whose mean is the measurement vector obtained from the estimated system, and whose variance is $R_{k}$. Due to this sampling step, this algorithm is often referred to as perturbed observations (or stochastic) EnKF. Although this procedure introduces an additional sampling error, previous work by Lawson and Hansen ${ }^{26}$ suggested it performs better in the presence of nonlinearities than its deterministic alternatives. 
The subscript $m \mid n$ is used to denote a random variable taken at time $t_{m}$ that has assimilated measurement data up to time $t_{n}$. It is worthy to note that one never needs to explicitly evaluate $\Sigma_{k}$ since it suffices to evaluate

$$
\Sigma_{k} H_{k}^{T}=\frac{1}{q-1} E_{k}^{s}\left(E_{k}^{o}\right)^{T} \text { and } H_{k} \Sigma_{k} H_{k}^{T}=\frac{1}{q-1} E_{k}^{o}\left(E_{k}^{o}\right)^{T} .
$$

Both the Particle Filter (PF) and the EnKF algorithms share the same forecast step, but their analysis steps are distinct. While in the PF the posterior PDF correspond to a linear combination of the prior ensemble whose weights are calculated using the Bayes' rule, the EnKF assign equal weights to all particles and correct the ensemble members themselves according to Kalman's update rule.

Because the KF assumes model linearity and that all probability distributions are Gaussian, optimality can only be expected as $q \rightarrow \infty$ provided these hypothesis hold. Under nonlinear dynamics, this sub-optimum filter can only be expected to provide estimates for the first two moments of a possible general state PDF. In fact, Le Gland ${ }^{27}$ demonstrated that in general the EnKF exhibit a $q^{-1 / 2}$ rate of convergence to the estimate asymptotic PDF. However, this limiting distributing may differ of the optimum filtering distribution since the optimum filtering solution may be multi-modal or exhibit other higher-order features in the presence on nonlinearities.

In a practical setting, the ensemble size $q$ required to guarantee the filter convergence will depend on the effective state space dimension (the dimensionality of the subspace/manifold in which the most relevant dynamics in confined) and on our ability of recognizing and sampling this meaningful part of the state space. ${ }^{23}$ Nevertheless, typical ensemble sizes do not exceed a few hundreds in view of the available computational power, and for such small ensemble sizes the EnKF exhibit some interesting characteristics.

First, since the correction added to each ensemble member during the analysis step is a combination of the forecast ensemble states, the analysis step operates only in the subspace spanned by the ensemble at hand. ${ }^{28}$ Therefore, the choice of initial ensemble can limit, at least initially, the effectiveness of the correction applied by the estimator to the ensemble members.

Also, small ensemble sizes lead to systematically underestimated error covariances. ${ }^{29}$ For a fixed measurement noise level, as the estimated covariance decreases, the weight given to the measurement data in the analysis step decreases and eventually becomes negligible. This phenomenon may lead to the divergence of the estimator and is known as covariance collapse in a reference to the fact that the particles collapse onto a single trajectory. The most commonly used fix for this issue is the covariance inflation (CI). This technique artificially increases the ensemble covariance in order to weight the measurement data more heavily. Kelly et al. $^{30}$ showed that for a large enough inflation, the boundedness of the EnKF can be guaranteed.

In general, the covariance inflation can be implemented as

$$
\hat{x}^{j}=\bar{x}+\beta\left(\hat{x}^{j}-\bar{x}\right)+\alpha^{j},
$$

where $\alpha^{j}$ is the additive covariance inflation vector that is usually drawn from a zero-mean normal distribution with covariance $S$, and $\beta$ is the multiplicative covariance inflation parameter. Whitaker and Hamill ${ }^{31}$ suggested that multiplicative inflation is especially useful in capturing the pernicious effects of the sampling errors associated with a small ensemble while additive inflation seems to be most effective in capturing sources of error that do not depend on the assimilation process such as the system modeling error. However, any practical implementation of an additive scheme requires prior knowledge of this modeling error (namely, a way of producing the matrix $S$ ).

The simplest multiplicative covariance inflation scheme is the one suggested by Anderson and Anderson ${ }^{22}$ (AA), in which $\beta$ is a scalar (typically, $\beta \in[1.005,1.05]$ ). This scheme corresponds to use $g_{k}\left(x_{k}\right)=\sqrt{\Sigma_{k}}$. After analyzing the effect of sampling errors introduced by limited-size ensembles, Sacher and Bartello ${ }^{32}$ concluded that more inflation is needed when observations lead to large corrections to the estimate. Later, Whitaker and Hamill ${ }^{31}$ proposed a inflation scheme termed relaxation-to-prior spread (RTPS). In this case, $\beta$ takes the form of the vector

$$
\beta_{i}=1+\theta\left(\frac{\sigma_{i}^{b}-\sigma_{i}^{a}}{\sigma_{i}^{a}}\right),
$$

where $\theta$ is a scalar (typically, $\theta \in[0.5,0.95]$ ), and $\sigma_{i}^{b}$ and $\sigma_{i}^{a}$ are, respectively, the prior and posterior ensemble standard deviation for the $i$-th state variable. Note that, because $\beta$ is now a vector, its multiplication with the perturbation vector $\left(\hat{x}^{j}-\bar{x}\right)$ must be performed component-wise.

Since the AA scheme results in particles that are linear combinations of the previous ones, they will naturally satisfy any kinematic constraints (boundary conditions). That is not the case with the RTPS 
scheme, which may yield con-conforming particles since each state variable is updated independently. In this particular implementation, the non-conforming part of the solutions are projected out using the numerical method discussed in the next section.

\section{Numerical Experiments}

The dynamics of the flow is represented by the discretized 2-D incompressible Navier-Stokes equations, and the simulations were carried out using the Immersed Boundary Projection Method (IBPM) ${ }^{33,34}$ enhanced by the Lattice Green's Function (LGF) formulation. ${ }^{35,36}$ The LGF formulation enforces exactly the freespace boundary condition at infinity even though the computation domain is restricted to the region of non-zero vorticity near the immersed body. The integrating factor approach is also used in conjunction with the LGF formulation to avoid the stiffness introduced by the viscous terms in the Navier-Stokes equation. Finally a half-explicit Runge-Kutta scheme, specially suitable to deal with systems of ODEs with algebraic constraints, is used as the time marching scheme.

In addition to dynamically update the ensemble members, this numerical solver is also used to generate the base solution and to collect the measurement data that was used for estimation purposes. This solution is regarded as the true state of the system which the estimator is supposed to track.

The initialization of the estimator plays a crucial role in its performance. Theoretically, in order to completely span a $n$-dimensional state space, one would need at least $n$ ensemble members. Instead, the initial ensemble is constructed using the improved sampling scheme proposed by Evensen. ${ }^{7}$ Starting from a dataset of snapshots of the base solution throughout 20 convective time units, the base mean flow $\bar{x}_{b}$ and the leading Proper Orthogonal Decomposition (POD) modes are computed. Then, the $q$ ensemble members are randomly sampled from the subspace spanned by the first $q$ POD modes of the data so that the ensemble average is $\bar{x}_{b}$ and the norm of ensemble covariance matrix matches the norm of the dataset covariance matrix. If the first few POD modes concentrate most of the energy of the flow, this sampling technique allows a more efficient distribution of the initial states.

In order to evaluate the performance of the estimator, the following metrics will be used:

- Estimate error: measures how the estimated state differs from the reference state:

$$
E_{x}=\frac{\left\|\bar{x}-x_{b}\right\|}{\left\|x_{b}\right\|} .
$$

- Ensemble state RMS: measures the uncertainty of the state estimate:

$$
R M S_{x}=\sqrt{\frac{1}{q-1} \sum_{i=1}^{q} \frac{\left\|x_{i}-\bar{x}\right\|^{2}}{\|\bar{x}\|^{2}}} .
$$

\section{III.A. Perfect model estimation}

The estimator performance will be significantly impacted by the choice of how to model and observe the tracked system. The definition of the predictive model must be a compromise between accuracy and cost. The predictive model should be accurate enough to alleviate the burden laid upon the error control techniques (stochastic forcing, covariance inflation, etc) while keeping the computational time expenditure controlled. On the other hand, the choice of what and where to measure not only limit the accuracy of the state/output estimates, but also determines if the estimation task is feasible at all (detectability).

In this subsection, we discuss how tuning the different estimation parameters affect the performance of the estimator in a perfect model framework. We show that the representers of the estimator (defined in Eq. 8) provide guidance on sensor placement.

\section{III.A.1. Flow past a circular cylinder}

The first test case is the canonical flow past a circular cylinder at $R e_{D}=100$. At this Reynolds number, the flow is still essentially two-dimensional ${ }^{37}$ and is characterized by the existence of coherent vortices in the wake known as the Karman vortex street. The dynamical system is deterministic, and, apart from synthetic noise added to the measurements, the only randomization is associated with the initial conditions. The goal is to use the EnKF to track the phase of the vortex street. 
The grid resolution is 25 points by diameter (which yields a system with about 15,000 degrees of freedom), and velocity components sampled at 14 equidistant points in the flowfield (see Fig. 1) are assimilated into the filtering process every 0.1 convective time units. The AA covariance inflation scheme with $\beta=1.05$ is used.

$\begin{array}{llll}0 & 0 & 0 & 0 \\ 0 & 0 & 0 & 0 \\ 0 & 0 & 0 & 0\end{array}$

Figure 1. Flow past a circular cylinder: location of the velocity measurement points in the flowfield.

Figure 2 shows how different choices of the initial ensemble impact the evolution of the estimate error when the ensemble size is held fixed at 24 members. In the first case, we initialize the flow field with zero-mean random numbers, while in the second case, we add random perturbations to the true mean flow. Finally, in the third case, we add the first 24 POD modes to the mean, but with coefficients randomized. In all cases, the ensemble initial variance matches the reference run variance. When the initial condition is restricted to the POD subspace, the estimator convergences within a few convective time units, whereas, unsurprisingly, the more random initial conditions take longer to converge. Physically speaking, the timescale associated with the domain size in the $\mathrm{x}$-direction is about 6 units, and the estimator must wash out the random initial condition over this time period while a transient takes place that leads to different realizations of the flow with distinct vortex shedding phases.

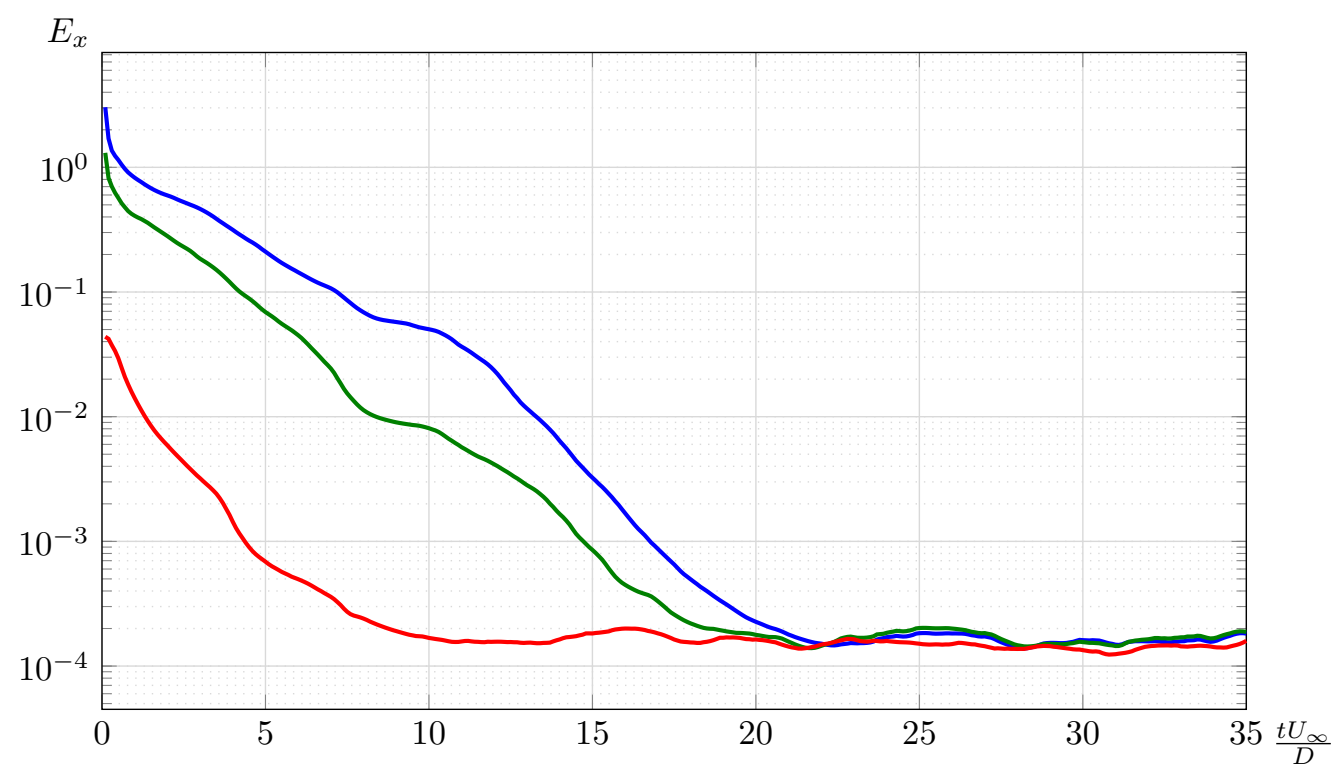

Figure 2. Effect of the choice of the initial ensemble on the estimator performance for the flow past a circular cylinder at $R e=100$ using velocity measurements. The ensemble has 24 members and the measurement error level is $R=$ $10^{-4} I_{p}$. ( $(-)$ corresponds to a zero-mean random initial condition, ( $\longrightarrow$ ) corresponds to the mean flow plus random perturbations and $(-)$ corresponds to the mean flow plus randomized leading POD modes.

Figure 3 presents the evolution of the state estimate error and ensemble RMS for different ensemble sizes. Provided that the dynamics is relatively sparse in the POD space and the most energetic subspace is 
captured by the initial ensemble, the transient behavior of the estimator appears to become independent of the ensemble size. This behavior indicates that the sampling error decays more rapidly than the expected $q^{-1 / 2}$ when a POD-based initialization is used. on the internal state is overestimated. As a consequence, error converges in a slower rate then when more ensemble members are used. However, if modeling errors were present, the increasing disregard for new external information coming from the measured data may lead the estimator to diverge.

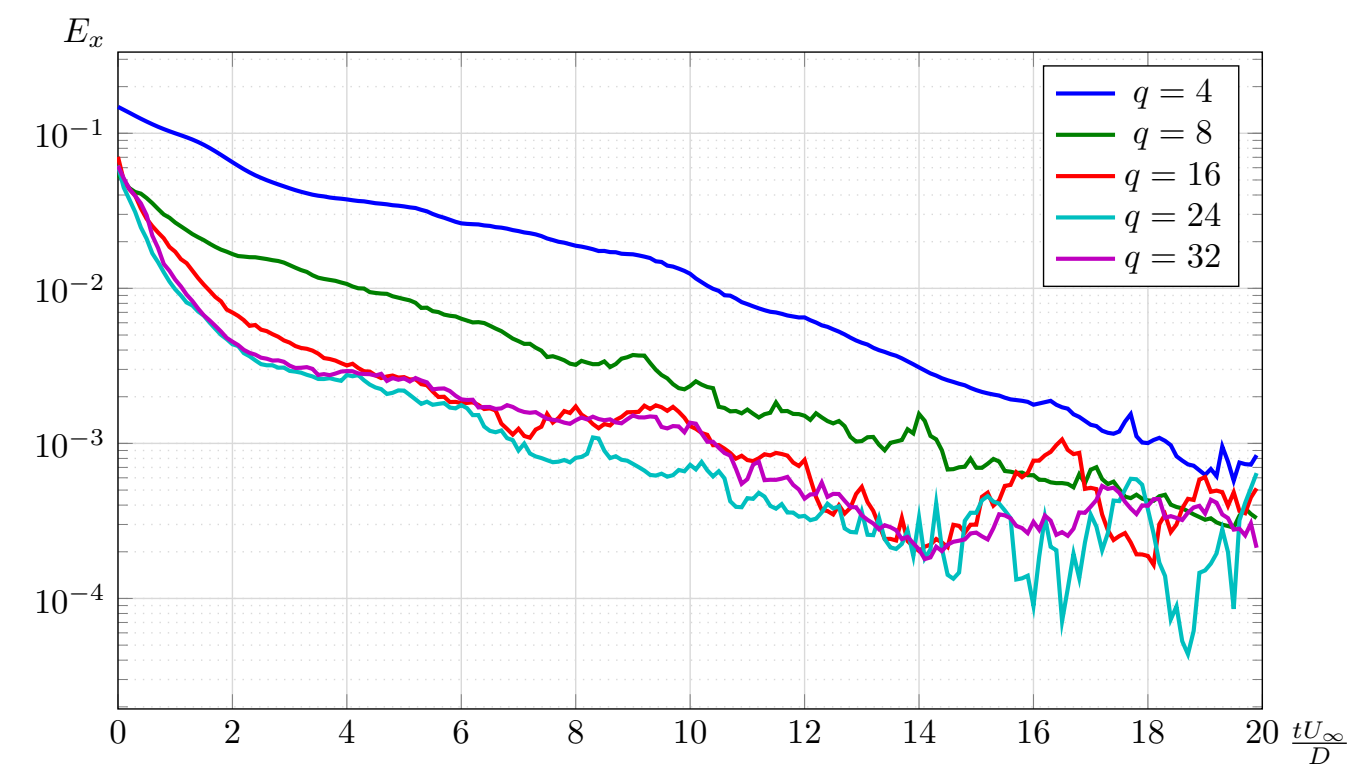

(a) State estimate error.

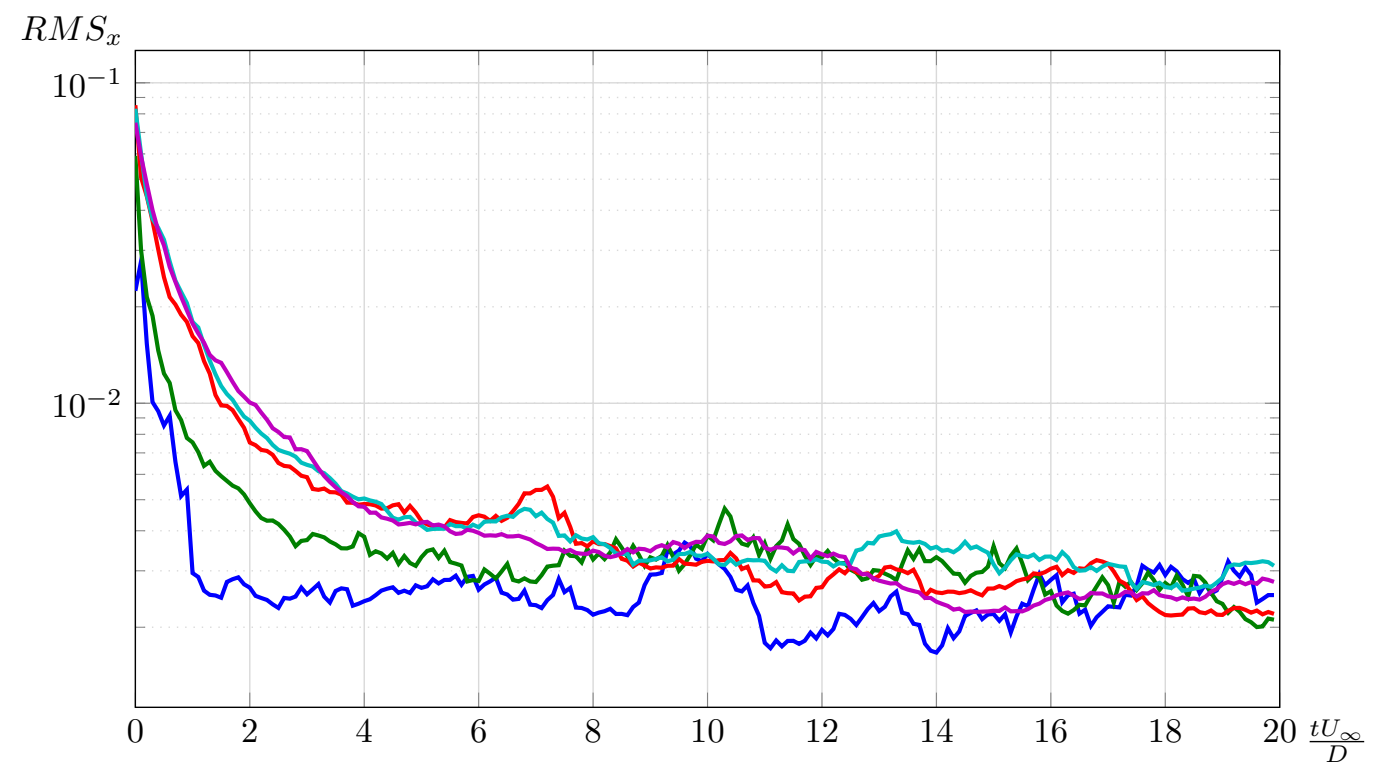

(b) Ensemble RMS.

Figure 3. Estimator performance for increasing ensemble sizes applied to the problem of the flow past a circular cylinder at $R e=100$ using velocity measurements. Ensemble size is set to 24 and $R=10^{-4} I_{p}$.

The measurement noise level also impacts the performance of the estimator, since the reliability of sensor data should be weighted against the internal state of the estimator. As the data assimilation proceeds, the estimated state uncertainty decreases and may reach an error level for which further corrections become negligible. Figure 4 shows how a lower noise level favors the estimator performance. Note that reducing the noise covariance level by two orders of magnitude reduces the ensemble RMS by one order of magnitude, as 
expected.

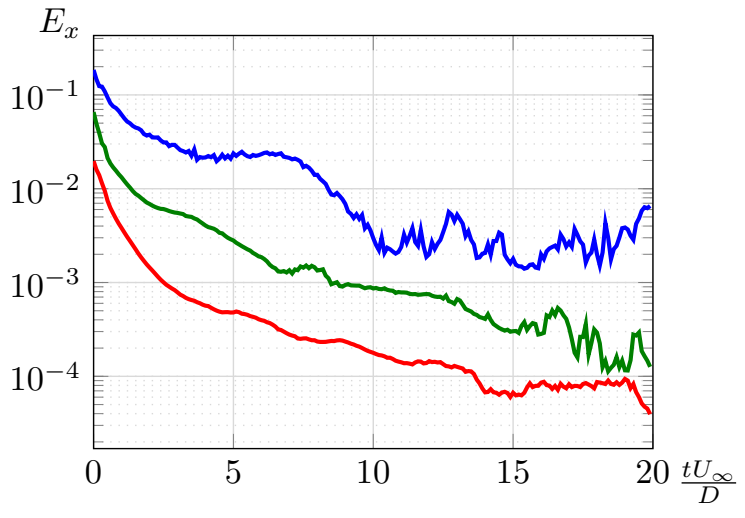

(a) State estimate error

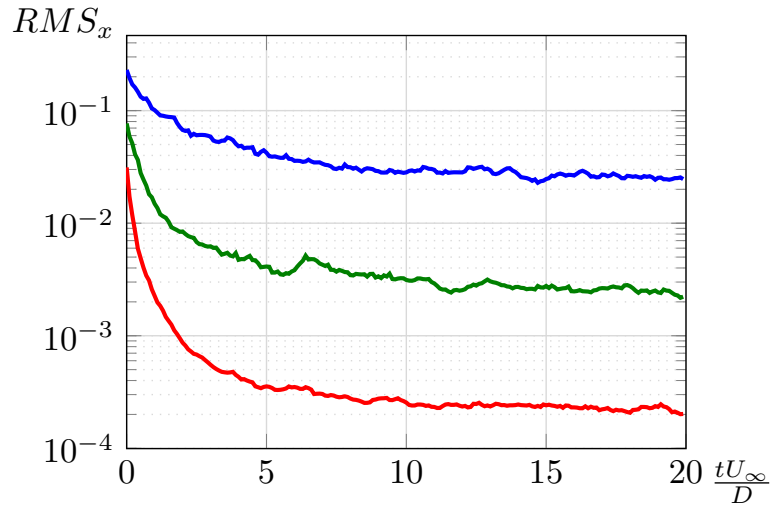

(b) Ensemble RMS

Figure 4. Estimator performance for distinct measurement noise levels applied to the problem of the flow past a circular cylinder at $R e=100$ using velocity measurements and 16 ensemble members. ( $(-)$, ( $(-)$ and $(\square)$ correspond, respectively, to measurement noise covariance matrices $R$ set to $10^{-2} I_{p}, 10^{-4} I_{p}$ and $10^{-6} I_{p}$.

Figure 5 shows the measurement influence fields (representers) for different measurement locations, as defined in 8 . The highest values are achieved for measurements taken about 2 diameters downstream of the cylinder. The rather obvious conclusion is that measurements taken at points where there is no variation in the solution amongst the ensemble members are useless. Interestingly, the region of highest influence is coincident with the so-called wavemaker region. ${ }^{38}$

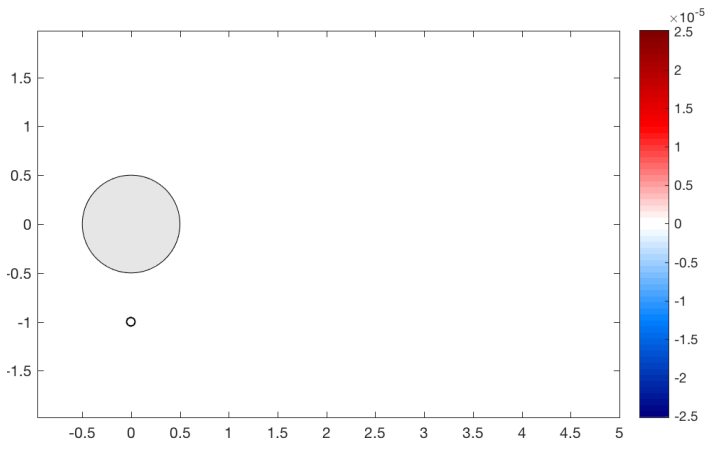

(a)

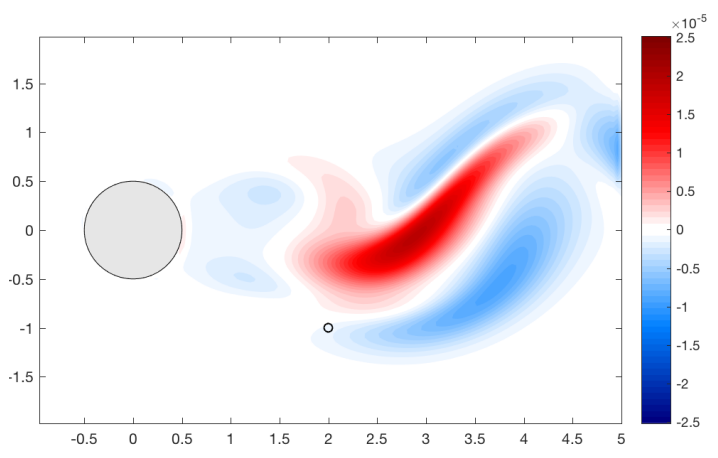

(c)

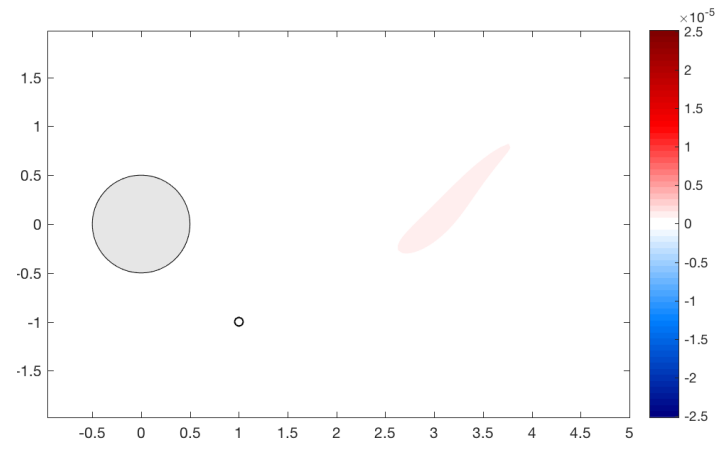

(b)

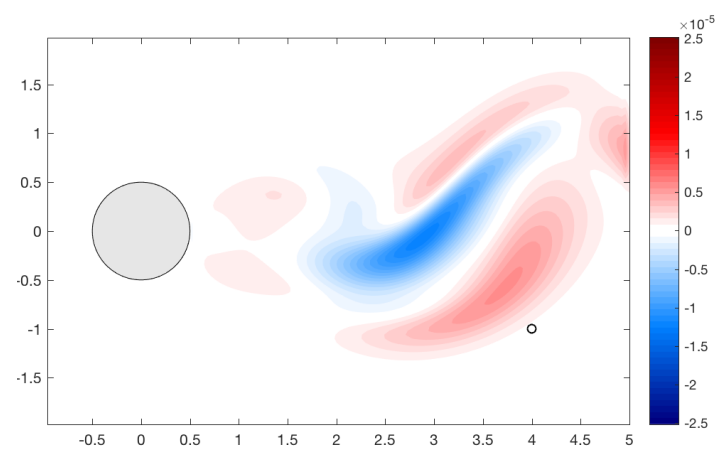

(d)

Figure 5. Measurement influence fields (representers) for the horizontal component of the fluid velocity at selected measurement locations. Ensemble size was 16 members and $R=10^{-4} I_{p}$. All the figures have the same contour levels. 


\section{III.A.2. Flow past a NACA 0009 airfoil at high angle of attack}

The second test case is closer to the motivation presented in the introduction section. The performance of the estimator is now analyzed when applied to the problem of the flow past a NACA 0009 at 30 degrees angle of attack and Reynolds number 200. The grid resolution is 50 points per chord and the pressure at distinct locations over the airfoil are taken as measurement data every 0.05 convective time units. The ensemble size was set to 24 in all cases. The estimator is able to track the vortex shedding phase with as few as ten measurements locations (see Fig. 6).

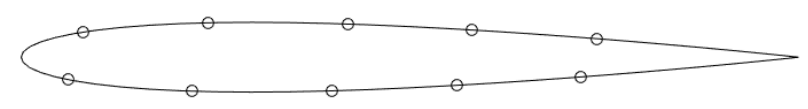

Figure 6. Location of the pressure measurement points over the surface of a NACA 0009 airfoil.

Figure 7 shows the estimated lift coefficient evolution with the RTPS scheme $(\theta=0.95)$. The behavior of the ensemble members can be analyzed using Eq. 9. Before the first analysis step, the variation of the ensemble predicted measurements $H_{k} \Sigma_{k} H_{k}^{T}$ is much larger than the measurement noise level $R_{k}$. As a consequence all ensemble members are strongly corrected toward the true solution in the first time step. At subsequent times, corrections are increasingly damped as the estimate converges to the true flow state and the measurement variance across the ensemble decreases.

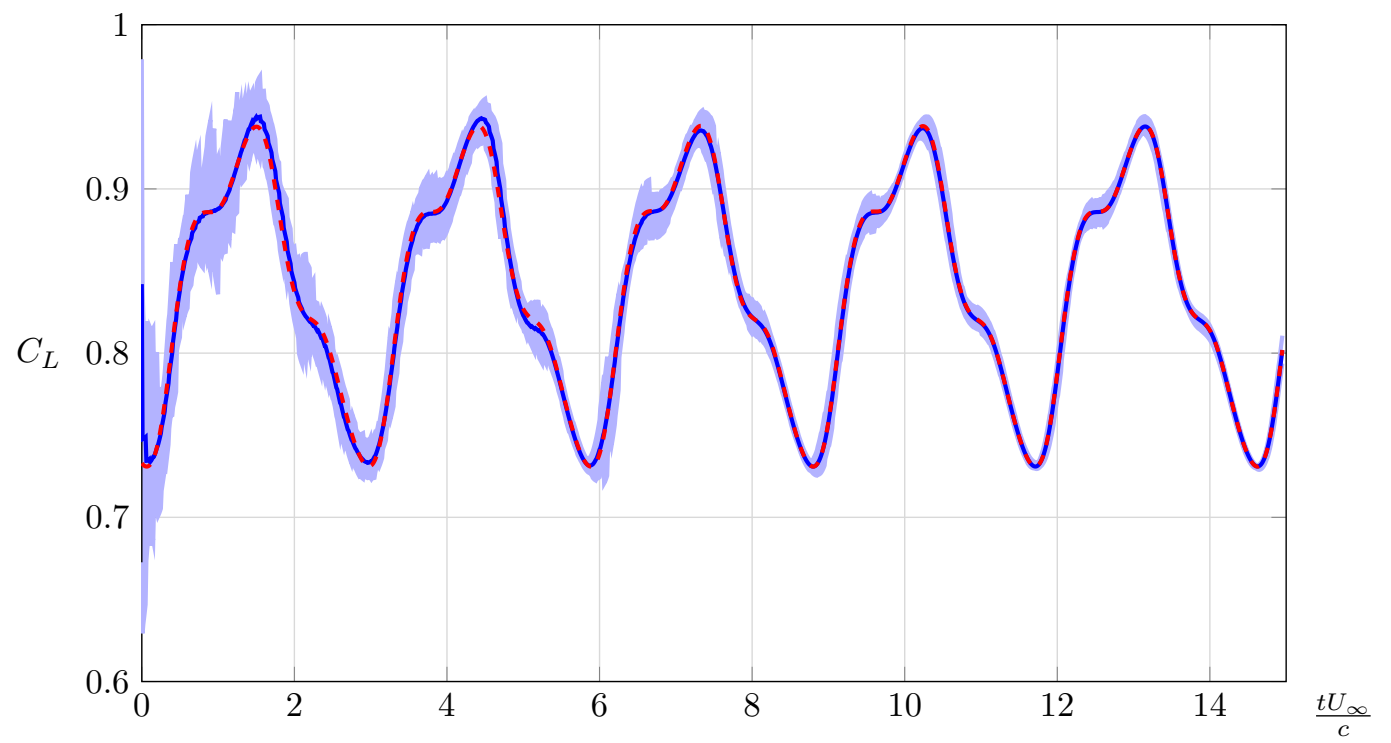

Figure 7. Estimated lift coefficient for the flow past a NACA 0009 airfoil using pressure measurements and the RTPS covariance inflation scheme $(\theta=0.95)$. The dashed line $(---)$, the solid line $(-)$, and the shaded area $(\square)$ represent, respectively, the reference solution, the EnKF estimate, and the ensemble min-max envelope $\left(R=10^{-4} I_{p}\right)$.

Figure 8 shows the estimate evolution for different choices of covariance inflation (CI) scheme. All schemes delay the decreasing trend of the ensemble variance and achieve a final estimate error smaller than the case with no inflation. In the AA scheme, a constant factor is used to artificially inflate the covariance and the error subspace spanned by the ensemble is preserved. As a result, corrections are similar to the ones that would have taken place in the absence of inflation but are more aggressive and take place for a longer period of time. The RTPS scheme, on the other hand, is more complex. The inflation magnitude is local and dependent upon the previous analysis step (for $\theta=1$, the forecast spread is recovered). Because of these characteristics, the RTPS scheme can in fact change the subspace spanned by the ensemble. This extra variability is possibly the reason for the distinct behavior at early times and may be even desirable for some applications. 


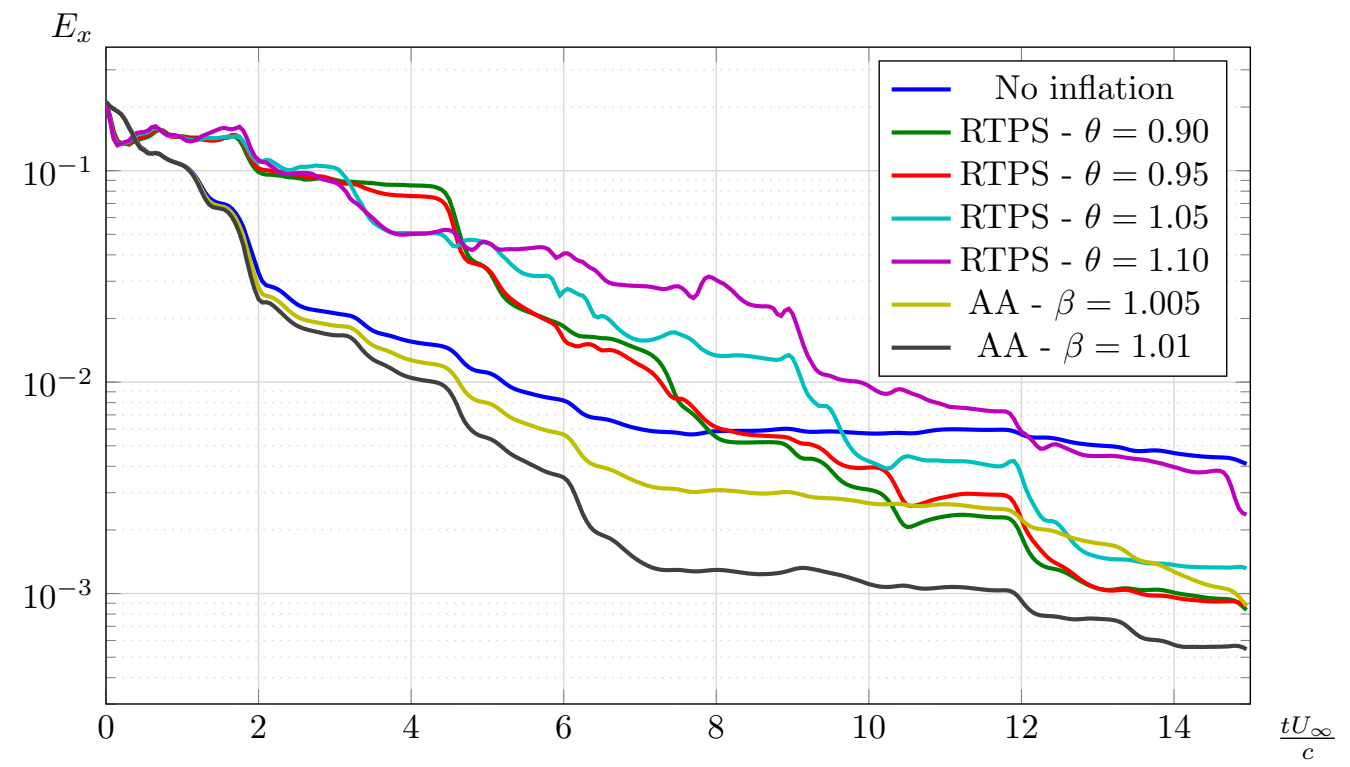

(a) State estimate error.

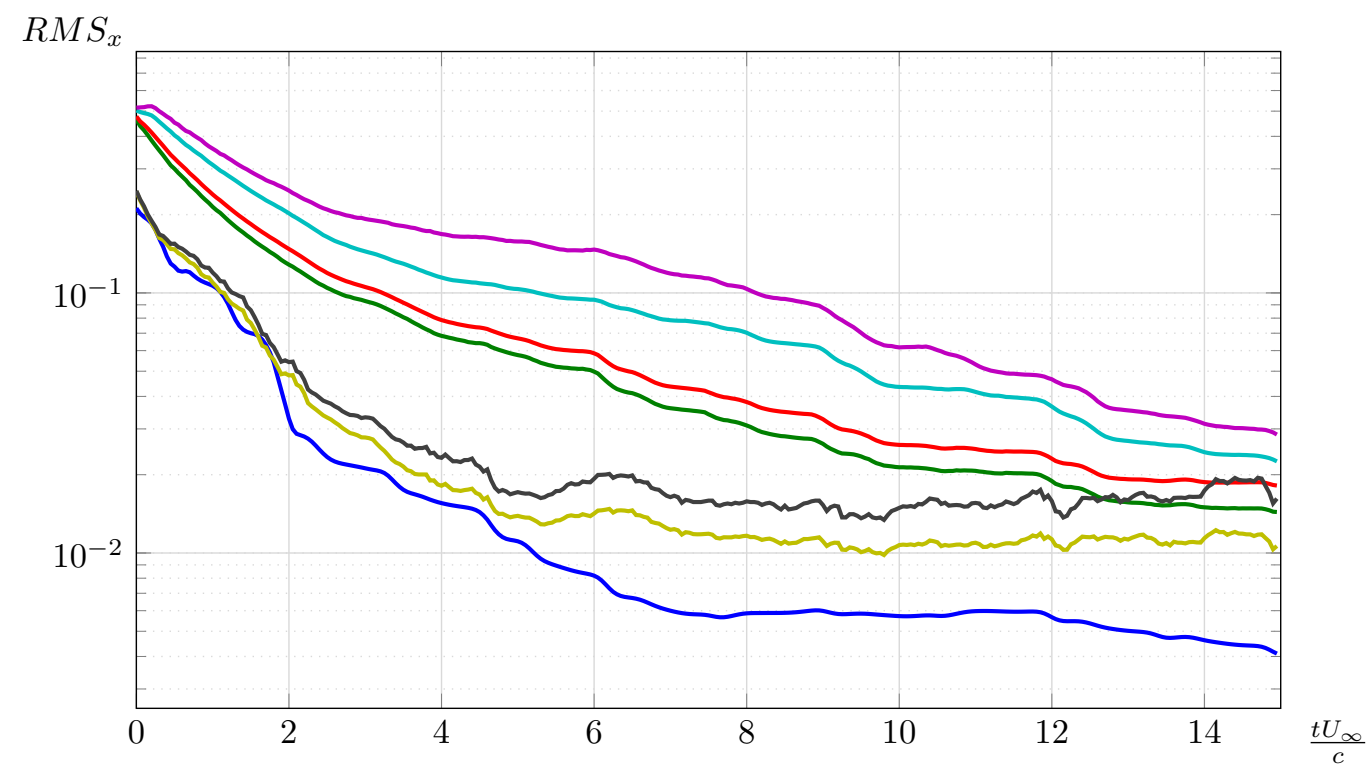

(b) Ensemble RMS.

Figure 8. Estimator performance for different multiplicative covariance inflation schemes applied to the flow past a NACA 0009 at high angle of attack. Ensemble size is 24 and the sensor noise level is set to $R=10^{-4}$.

\section{III.B. Estimation in the presence of modeling errors}

In practical applications, models are imperfect and there are aspects of the dynamics that are not represented in the estimator. As discussed in section II, additive covariance inflation should be the preferred way to mitigate these errors when their source is unknown or hard to be directly modeled. This approach is based on the assumption that their effect can be represented by a Gaussian-distributed stochastic forcing with a prescribed mean (often referred as the model bias) and covariance $S$.

In fields like meteorology, several researchers ${ }^{39,40}$ have proposed error covariance models whose corresponding parameters can be tuned on-line. These models often rely on some low-order representation of these matrices (a popular choice is to use the slow modes of the forecast model). When a suitable representation of these errors in terms of bias and associated covariance is not available, the implementation of this approach becomes impractical. In the absence of a preferential subspace that a low-order representation of the model 
error covariance matrix would provide, a consistent sampling would require a large ensemble.

Instead, recall that inflating the ensemble covariance is equivalent to weighting the actual measurements to the detriment of the ones predicted by the estimator internal state, ${ }^{30}$ allowing more aggressive corrections to the state and enhancing the estimator responsiveness. All corrections, however, will be confined to subspace spanned by the prior ensemble. In the absence of system noise (either explicitly or in the form of additive CI), the only mechanisms that introduce variability to this subspace are the estimator dynamic model and, perhaps, the multiplicative CI. Among the two schemes that were used before, only the RTPS is able of such and it is, therefore, the chosen scheme for this problem.

The following examples are built upon the basic formulation proposed in section III.A.2. Two types of perturbations are introduced to the free stream velocity of the nature run, affecting both the forecast and measurement evaluations.

\section{III.B.1. Random free stream perturbation}

In this test case, the reference solution is perturbed by setting the free stream velocity to

$$
U_{\infty}(t)=1+0.1 \mathcal{F}_{1}(\xi(t))
$$

where $\xi(t)$ is a random Gaussian noise sequence and $\mathcal{F}_{1}(\cdot)$ is a 8th-order Butterworth filter with a unitary cutoff reduced frequency $\left(f c / U_{r e f}\right)$. Figure 9 shows an example of a perturbation sequence obtained using this procedure.

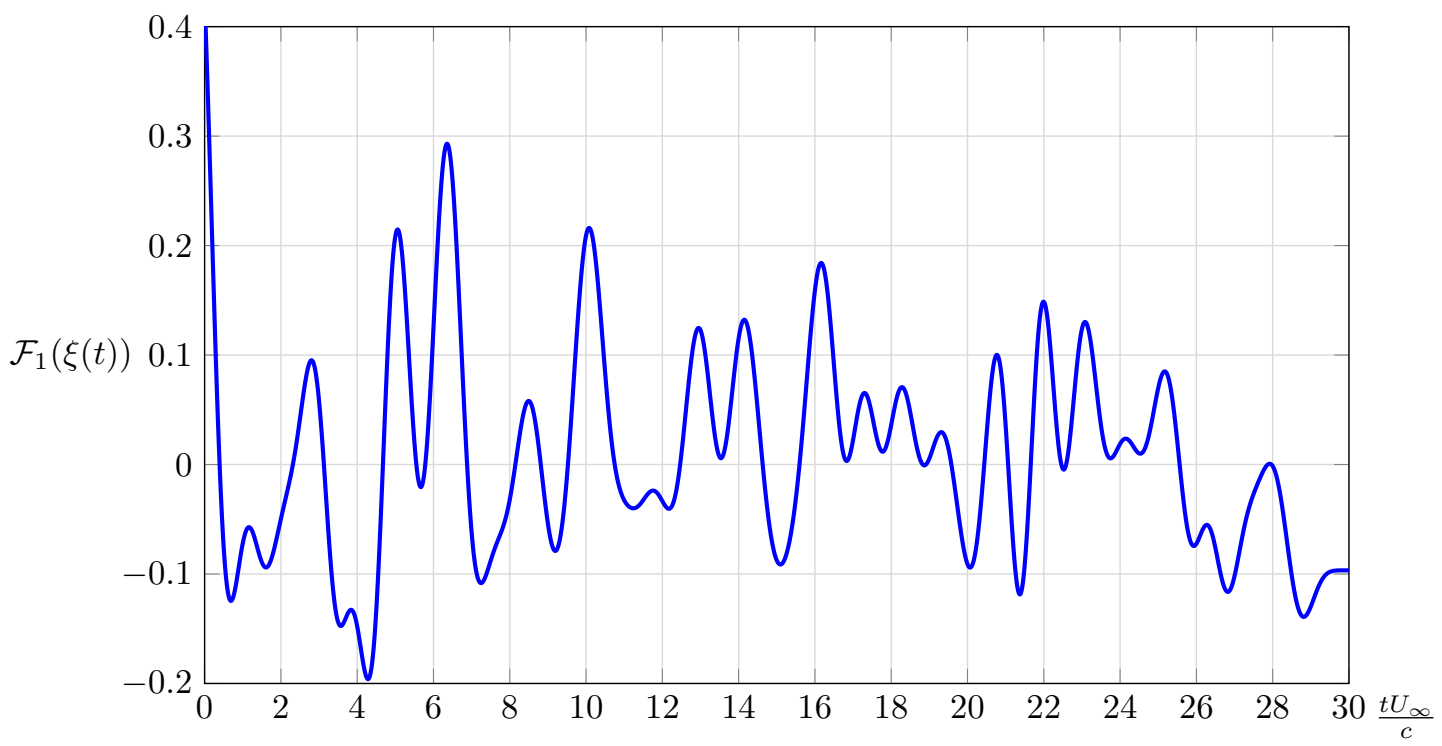

Figure 9. Example of the velocity perturbations introduced to the free stream using the 8th-order Butterworth filter with a unitary cutoff reduced frequency.

The estimator dynamic model is made to be agnostic to the presence of the perturbations and enforces a unitary boundary condition at all times. Following the aforementioned approach, no modification to the dynamic model is made other than increasing the multiplicative covariance inflation parameter. Figure 10 shows the ensemble lift coefficient evolution for two different choices of covariance inflation magnitude and ensemble size. Increasing the former leads to a more aggressive correction behavior which tends to yield noisier estimates. To some extent, this tendency can be mitigated with a larger ensemble size. A combination of both strategies usually leads to enhanced tracking capabilities. 


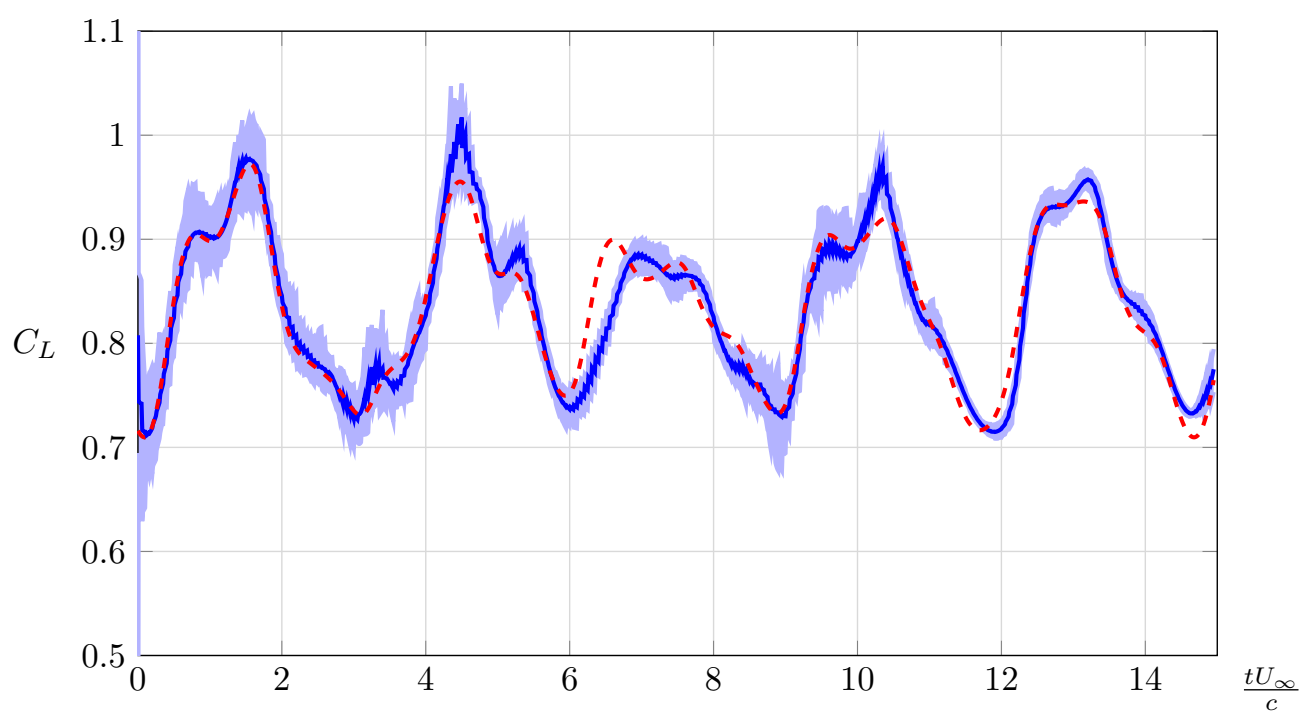

(a) Ensemble size 24 and $\theta=1.05$.

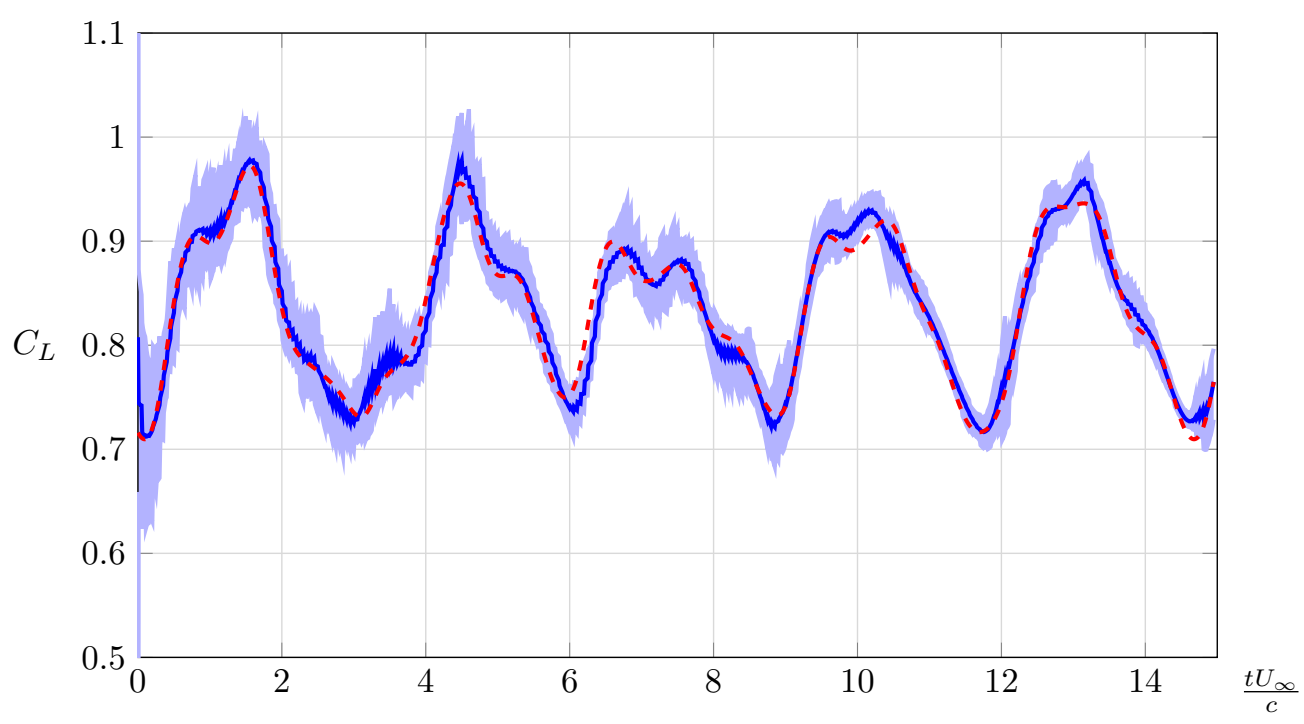

(b) Ensemble size 48 and $\theta=1.20$

Figure 10. Estimated lift coefficient for airfoil with randomized freestream velocity using the RTPS covariance inflation scheme $\left(R=10^{-4} I_{p}\right)$. The dashed line $(---)$, the solid line $(\square)$, and the shaded area $(\square)$ represent, respectively, the reference solution, the EnKF estimate, and the ensemble min-max envelope.

\section{III.B.2. Prescribed free stream perturbation}

In this last test case, the free stream velocity is perturbed in a deterministic way

$$
U_{\infty}(t)=1-\frac{\alpha}{2} \operatorname{erfc}\left(-\frac{\sqrt{\pi} \beta}{\alpha}\left(t-t_{0}\right)\right)
$$

where $\operatorname{erfc}(\mathrm{t})$ is the complementary error function, $\alpha$ is the saturation value, $\beta$ is the slope at $t=t_{0}$, the reference time at which the perturbation reaches half of its saturation value. In this particular setup, the following values were used: $\alpha=0.25, t_{0}=1000$ in grid units, and $\beta=3.7510^{-4}$.

For this example, the goal is to equip the estimator to be able to track the free stream perturbation. The free stream velocity is modeled as exhibiting a linear behavior between two consecutive analysis steps, such that the state vector is then augmented with the inclusion of the free stream perturbation and its derivative. The dynamic model for them is a simple integrator with the derivative being propagated as a constant. This 
approach of simultaneously estimate both state and parameters is often referred to as joint estimation, and has the advantage of taking into account the cross-correlation between the state and the parameters.

Figure 11 shows some interesting features of the EnKF. Because the IB forces acting on the body are a function of the vorticity field, and the free stream velocity and acceleration, the lift (the sum of the vertical components of these forces) is a good global performance indicator for this estimation problem. For early times, the $C_{L}$ ensemble variance is larger than the perfect model case due to extra degree of uncertainty added by the presence of a perturbation of unknown magnitude, but the estimator is able to obtain the right phase after just 2 convective time units and most of the perturbation tracking is performed while the estimator has already a good estimate for the state of the system.

Figure 12 shows how the parameters estimate evolves with a increasing inflation magnitude. For early times, the poorer estimates are consistent with the transient behavior that was observed in Fig. 11. The impact of the covariance inflation parameter is more pronounced in the acceleration, in which higher values lead to a better overall tracking but noisier initial estimates.

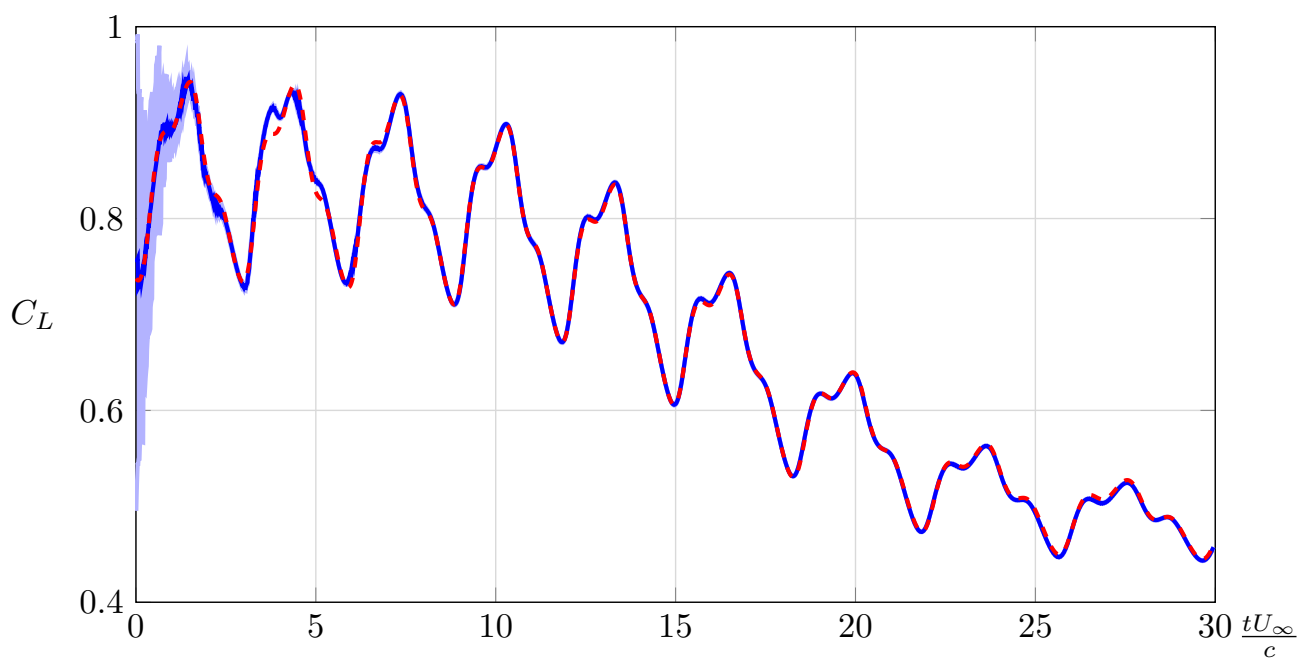

Figure 11. Estimated lift coefficient for decelerating airfoil using the RTPS $(\theta=0.9)$ covariance inflation scheme and $R=10^{-6} I_{p}$. The dashed line $(---)$, the solid line ( -$)$, and the shaded area ( $)$ represent, respectively, the reference solution, the EnKF estimate, and the ensemble min-max envelope.

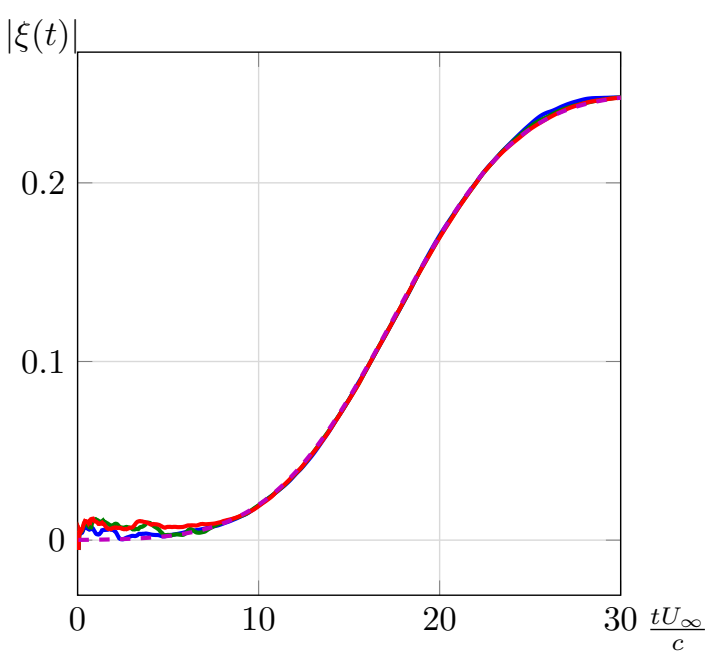

(a) Free stream velocity perturbation.

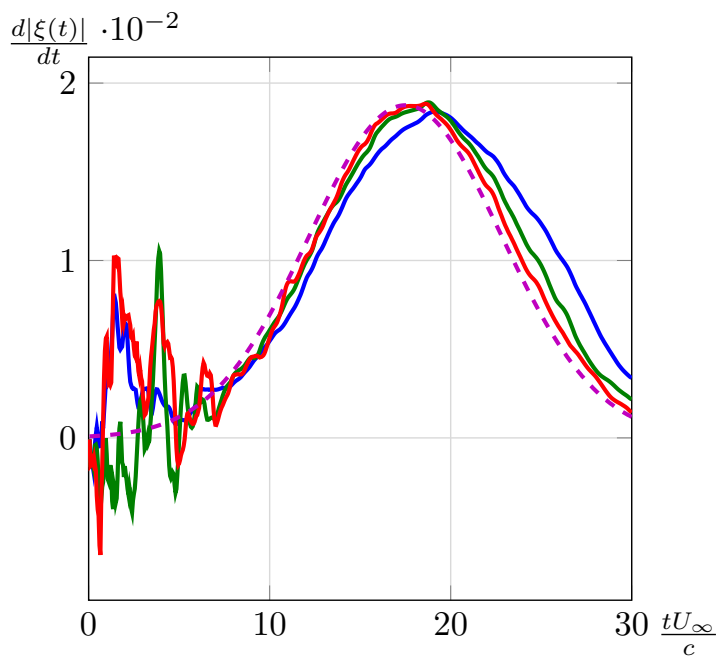

(b) Free stream velocity perturbation derivative.

Figure 12. Joint parameter/state estimation for decelerating airfoil. The measurement noise level is set to $R=10^{-6} I_{p}$. The dashed line (-- ) corresponds to the actual perturbation, and the solid lines represent the estimated values for different RTPS inflation levels: $\theta=0.80(-), \theta=0.90(-)$ and $\theta=0.95((-)$. 


\section{Concluding remarks}

In this paper, a dual experiment methodology, in which the numerical algorithm as both the estimator and as a surrogate for the measurements, was used to investigate the performance of an EnKF-based estimator for simple aerodynamic problems. It was demonstrated to provide an estimation framework in which one can combine the fidelity and robustness of using high-dimensional representation of fluid systems as the predictive model, with the computational efficiency of a low-order representation of the covariance matrix.

A reduced number of either velocity or pressure measurements were successfully used to estimate the phase of the vortex shedding, and thus the corresponding forces. In terms of performance, global (velocity in the wake) measurements are more efficient in constraining the vorticity within the domain. Estimation based on local (pressure on the surface) measurements takes longer to achieve the same state error level. However, using pressure measurements makes the estimator more responsive in tracking the forces acting on the body, which is especially desirable when free stream perturbations are present.

For dynamical systems whose relevant features are restricted to a relatively small subspace/manifold of the state space, results indicates that small ensemble sizes may be enough to provide a suitable representation of the covariance matrix and lead to an asymptotic behavior of the estimator dynamics.

The analysis of the measurement influence fields (representers) provided interesting a posteriori information about where measurements are more effective to the filtering process, and can provide useful guidance on sensor placement. In the cylinder case, the region of higher influence seems to be related to the wavemaker region predicted by structural sensitivity analysis.

Multiplicative covariance inflation was used with two objectives: delaying the covariance collapse induced by the reduced ensemble size, and enabling a larger weighting of the estimator towards the measurements taken from the reference solution when unmodeled forecast errors are present. In Kalman's approach to the analysis step, the estimated system covariance is bound to decrease until it reaches a level (determined by the measurement noise level) in which new measurements have little impact. The distinct behavior shown in Fig. 4 is an example to the dynamic balance that comes into play as the reliability of internal state is weighted against the measurement uncertainty.

Alternatively, when the source of the forecast errors is known, parametric modeling can be used to upgrade the estimator model. Parameters and state can then be jointly estimated by using a augmented state-vector approach.

\section{Acknowledgments}

This work has been supported in part by a grant from AFOSR (FA9550-14-1-0328) with Dr. Douglas Smith as program manager. A.F.C. da Silva would like to thank the Ministry of Education of Brazil (Capes Foundation) for its support through a Science without Borders scholarship (Grant number BEX 12966/134). The authors also acknowledge Prof. Andrew Stuart, Prof. David Williams and Prof. Jeff Eldredge for helpful discussions of this work.

\section{References}

\footnotetext{
${ }^{1}$ Kalman, R. E. and Bucy, R. S., "New Results in Linear Filtering and Prediction Theory," Journal of Basic Engineering, Vol. 83, No. 1, 1961, pp. 95-108.

${ }^{2}$ Hayase, T., Nisugi, K., and Shirai, A., "Numerical Realization for Analysis of Real Flows by Integrating Computation and Measurement," International Journal for Numerical Methods in Fluids, Vol. 47, No. 6-7, 2005, pp. 543-559.

${ }^{3}$ Ahuja, S. and Rowley, C. W., "Feedback Control of Unstable Steady States of Flow Past a Flat Plate Using Reduced-Order Estimators," Journal of Fluid Mechanics, Vol. 645, Feb 2010, pp. 447.

${ }^{4}$ Flinois, T. L. B. and Morgans, A. S., "Feedback Control of Unstable Flows: A Direct Modelling Approach Using the Eigensystem Realisation Algorithm," Journal of Fluid Mechanics, Vol. 793, 2016, pp. 41.

${ }^{5}$ Fukumori, I. and Malanotte-Rizzoli, P., "An Approximate Kalman Filter for Ocean Data Assimilation: An Example With an Idealized Gulf Stream Model," Journal of Geophysical Research: Oceans, Vol. 100, No. C4, 1995, pp. 6777-6793.

${ }^{6}$ Suzuki, T., "Reduced-Order Kalman-Filtered Hybrid Simulation Combining Particle Tracking Velocimetry and Direct Numerical Simulation," Journal of Fluid Mechanics, Vol. 709, 2012, pp. 249-288.

${ }^{7}$ Evensen, G., Data Assimilation: The Ensemble Kalman Filter, Springer Science \& Business Media, 2009.

${ }^{8}$ Kalnay, E., Atmospheric Modeling, Data Assimilation and Predictability, Cambridge University Press, 2003.

${ }^{9}$ Colburn, C. H., Cessna, J. B., and Bewley, T. R., "State Estimation in Wall-Bounded Flow Systems. Part 3. the Ensemble Kalman Filter," Journal of Fluid Mechanics, Vol. 682, Aug 2011, pp. 289-303.
} 
${ }^{10}$ Kikuchi, R., Misaka, T., and Obayashi, S., "Assessment of Probability Density Function Based on POD Reduced-Order Model for Ensemble-Based Data Assimilation," Fluid Dynamics Research, Vol. 47, No. 5, 2015, pp. 051403.

${ }^{11}$ Lorenc, A. C., "Analysis Methods for Numerical Weather Prediction," Quarterly Journal of the Royal Meteorological Society, Vol. 112, No. 474, 1986, pp. 1177-1194.

${ }^{12}$ Stengel, R. F., Optimal Control and Estimation, Courier Corporation, 2012.

${ }^{13}$ Gillijns, S., Mendoza, O. B., Chandrasekar, J., De Moor, B. L. R., Bernstein, D. S., and Ridley, A., "What Is the Ensemble Kalman Filter and How Well Does It Work?" Proceedings of the 2006 American Control Conference, IEEE, Jun 2006, pp. 4448-4453.

${ }^{14}$ Gelb, A., Applied Optimal Estimation, MIT press, 1974.

${ }^{15}$ Julier, S. J. and Uhlmann, J. K., "Unscented Filtering and Nonlinear Estimation," Proceedings of the IEEE, Vol. 92, No. 3, 2004, pp. 401-422.

${ }^{16}$ Wan, E. A. and Van Der Merwe, R., "The Unscented Kalman Filter for Nonlinear Estimation," Adaptive Systems for Signal Processing, Communications, and Control Symposium 2000. AS-SPCC. The IEEE 2000, IEEE, 2000, pp. 153-158.

${ }^{17}$ van Leeuwen, P. J., "Particle Filtering in Geophysical Systems," Monthly Weather Review, Vol. 137, No. 12, 2009, pp. 4089-4114.

${ }^{18}$ Bellman, R. E., Dynamic Programming, Dover Publications, Incorporated, 2003.

${ }^{19}$ Evensen, G., "Sequential Data Assimilation With a Nonlinear Quasi-Geostrophic Model Using Monte Carlo Methods to Forecast Error Statistics," Journal of Geophysical Research: Oceans, Vol. 99, No. C5, 1994, pp. 10143-10162.

${ }^{20}$ Bengtsson, T., Snyder, C., and Nychka, D., "Toward a Nonlinear Ensemble Filter for High-Dimensional Systems," Journal of Geophysical Research: Atmospheres, Vol. 108, No. D24, 2003.

${ }^{21}$ Evensen, G., "Sampling Strategies and Square Root Analysis Schemes for the EnKF," Ocean Dynamics, Vol. 54, No. 6, 2004, pp. 539-560.

${ }^{22}$ Anderson, J. L. and Anderson, S. L., "A Monte Carlo Implementation of the Nonlinear Filtering Problem to Produce Ensemble Assimilations and Forecasts," Monthly Weather Review, Vol. 127, No. 12, 1999, pp. 2741-2758.

${ }^{23}$ Papadakis, N., Mémin, É., Cuzol, A., and Gengembre, N., "Data Assimilation with the Weighted Ensemble Kalman Filter," Tellus A, Vol. 62, No. 5, 2010, pp. 673-697.

${ }^{24}$ Nerger, L., Hiller, W., and Schröter, J., "A Comparison of Error Subspace Kalman Filters," Tellus A, Vol. 57, No. 5, 2005, pp. 715-735.

${ }^{25}$ Evensen, G. and Van Leeuwen, P. J., "Assimilation of Geosat Altimeter Data for the Agulhas Current Using the Ensemble Kalman Filter With a Quasigeostrophic Model," Monthly Weather Review, Vol. 124, No. 1, 1996, pp. 85-96.

${ }^{26}$ Lawson, W. G. and Hansen, J. A., "Implications of Stochastic and Deterministic Filters as Ensemble-Based Data Assimilation Methods in Varying Regimes of Error Growth," Monthly Weather Review, Vol. 132, No. 8, 2004, pp. $1966-1981$.

${ }^{27}$ Le Gland, F., Monbet, V., and Tran, V.-D., "Large Sample Asymptotics for the Ensemble Kalman Filter," Research Report RR-7014, Institut National de Recherche en Informatique et en Automatique (INRIA), 2009.

${ }^{28}$ Evensen, G., "The Ensemble Kalman Filter: Theoretical Formulation and Practical Implementation," Ocean dynamics, Vol. 53, No. 4, 2003, pp. 343-367.

${ }^{29}$ van Leeuwen, P. J., "Comment on Data Assimilation Using an Ensemble Kalman Filter Technique," Monthly Weather Review, Vol. 127, No. 6, 1999, pp. 1374-1377.

${ }^{30}$ Kelly, D., Law, K., and Stuart, A. M., "Well-Posedness and Accuracy of the Ensemble Kalman Filter in Discrete and Continuous Time," Nonlinearity, Vol. 27, No. 10, 2014, pp. 2579.

${ }^{31}$ Whitaker, J. S. and Hamill, T. M., "Evaluating Methods to Account for System Errors in Ensemble Data Assimilation," Monthly Weather Review, Vol. 140, No. 9, 2012, pp. 3078-3089.

${ }^{32}$ Sacher, W. and Bartello, P., "Sampling Errors in Ensemble Kalman Filtering. Part I: Theory," Monthly Weather Review, Vol. 136, No. 8, 2008, pp. 3035-3049.

${ }^{33}$ Taira, K. and Colonius, T., "The Immersed Boundary Method: A Projection Approach," Journal of Computational Physics, Vol. 225, No. 2, 2007, pp. 2118-2137.

${ }^{34}$ Colonius, T. and Taira, K., "A Fast Immersed Boundary Method Using a Nullspace Approach and Multi-Domain FarField Boundary Conditions," Computer Methods in Applied Mechanics and Engineering, Vol. 197, No. 25, 2008, pp. $2131-2146$.

${ }^{35}$ Liska, S. and Colonius, T., "A Parallel Fast Multipole Method for Elliptic Difference Equations," Journal of Computational Physics, Vol. 278, 2014, pp. 76-91.

${ }^{36}$ Liska, S. and Colonius, T., "A Fast Immersed Boundary Method for External Incompressible Viscous Flows Using Lattice Green's Functions," Journal of Computational Physics, Vol. 331, 2017, pp. 257-279.

${ }^{37}$ Williamson, C. H. K., "The Existence of Two Stages in the Transition to Threedimensionality of a Cylinder Wake," Physics of Fluids, Vol. 31, No. 11, 1988, pp. 3165-3168.

${ }^{38}$ Giannetti, F. and Luchini, P., "Structural Sensitivity of the First Instability of the Cylinder Wake," Journal of Fluid Mechanics, Vol. 581, Jun 2007, pp. 167197.

${ }^{39}$ Dee, D. P., "On-line Estimation of Error Covariance Parameters for Atmospheric Data Assimilation," Monthly Weather Review, Vol. 123, No. 4, 1995, pp. 1128-1145.

${ }^{40}$ Cohn, S. E. and Parrish, D. F., "The Behavior of Forecast Error Covariances for a Kalman Filter in Two Dimensions," Monthly Weather Review, Vol. 119, No. 8, 1991, pp. 1757-1785. 